\title{
Real-time Monitoring of Patients with Diabetes Using Software Applications - Proposing a Conceptual Model
}

\author{
Iustina-Cristina COSTEA-MARCU \\ Politehnica University, Bucharest, Romania \\ costeamarcuiustina@gmail.com \\ Gheorghe MILITARU \\ Politehnica University, Bucharest, Romania \\ ghmilitaru.militaru@gmail.com \\ Mihaela-Rodica GANCIU \\ Energomontaj S.A., Bucharest, Romania \\ mihaella.ganciu@gmail.com
}

\begin{abstract}
The rate of diabetes has increased in proportion to that of obesity and thus is a topical issue that is carefully studied and for which various IT solutions are proposed to facilitate the treatment and monitoring of patients. There are many applications that can be used by those diagnosed with diabetes, those with various specifications useful in ensuring a healthy lifestyle, reminders for testing and nutritional journals. This paper highlights a brief analysis of existing applications for monitoring patients with diabetes and proposes a useful computer application in collaboration between the specialist, patient and laboratory physician. Many people do not have access to specialized medical services (especially those living in rural areas, in isolated areas) and at the same time, the current context of the pandemic proves the need for certain technological solutions for monitoring patients at home.
\end{abstract}

Keywords: diabetes, medical application, real-time monitoring, home care.

\section{Introduction}

Diabetes is a topical issue given that the rate of diabetes has increased in proportion to that of obesity. The statistics show that the prevalence of diabetes is two times higher in overweight people compared to the normal weight and seven times higher in the obese. According to statistics provided by the International Diabetes Federation, in 2019, there were 463 million adults (20-79 years) worldwide with diabetes (International Diabetes Federation, 2020).

There are three types of diabetes: type 1 diabetes, type 2 diabetes and gestational diabetes. Type 1 diabetes is observed when the pancreas does not produce insulin, and type 2 diabetes occurs when the pancreas does not produce enough insulin or it cannot be processed. Gestational diabetes occurs in pregnant women when insulin is less effective. More than 20 million live births are affected by diabetes during pregnancy (International Diabetes Federation, 2020).

The paper presents an analysis of current solutions for home monitoring of patients with diabetes and also presents an application concept that can be used in monitoring any patient with diabetes in real time. Health informatization brings a series of tools that come in order to provide consistent support to this field in the form of various systems and services. The desired result from the integration of technologies in this field is to increase the quality of services, reduce their costs, real-time monitoring and many other benefits for all in this field (patients, doctors, private or public medical institutions). 


\section{Literature review}

Diabetes is a metabolic imbalance characterized by an increased concentration of glucose in the blood. In order for glucose to enter cells, it needs the presence of insulin. Insulin is a hormone produced by the pancreas. When we eat it is automatically secreted in the required amount. But in people with diabetes, the pancreas either produces less insulin or does not produce it at all, or the cells do not respond properly to secreted insulin (Campbell et al., 2021).

Diabetes can be kept under control through careful monitoring of diet and weight and

PICBE |

34 exercise, as a supplement to medical treatment. Diagnosis of patients with diabetes is based on tests: determination of glucose in the urine (normally it is 0 but if the blood sugar exceeds $180 \mathrm{mg}$, glucose also appears in the urine), measurement of uric acid, measurement of urea, measurement of albumin, cholesterol analysis ( the body tries to replace the lack of glucose with fats and therefore the blood of diabetics is fatty).

Monitoring patients with diabetes is primarily about controlling blood sugar. Regular blood glucose testing helps evaluate the effectiveness of diet, physical activity and drug treatment. The $\mathrm{Hb} \mathrm{A} 1 \mathrm{c}$ test (known as glycated hemoglobin) measures the average blood sugar over a period of time by collecting a specific component of the red blood cell. HbAlc is recommended for use in diagnosing diabetes by the American Diabetes Association (Arnold et al., 2020). Blood glucose is measured using a glucometer. Another parameter that should be monitored in diabetics is blood pressure. In most cases, people with diabetes suffer from high blood pressure and in this case the risk of cardiovascular disease or complications typical of diabetes increases. Cholesterol levels and weight should also be monitored (Petrie et al., 2018).

The American Diabetes Association (ADA2020) has presented a study demonstrating the importance of using telemedicine, more specifically the telemonitoring component in patients with poorly controlled type 2 diabetes who live in rural areas and have limited access to specialized medical services. Diabetes technology, combined with education and monitoring, can improve the lives and health of people with diabetes (American Diabetes Association, 2019). Bluetooth Low Energy (BLE) based sensors can be considered as a solution for monitoring personal data of vital signs. The number of portable BLE sensors, mobile devices and other connected things is increasing significantly (Alfian et al., 2018).

There are several types of applications (Doyle, 2020; Jimenez et al.,2019) that can be useful in treating diabetes, in various categories: calorie calculation or nutritional suggestions (eg: Go Meal, My Fitness, Diabetes: M, Beat Diabetes, Calorie Counter), insulin dosing (eg: Insulin Dose Calculator Pro, Insulin Calculator, Diabetes Personal Calculator), glucose monitoring (eg Diabetes Net Diary, MySugr, Blood Sugar Monitor by Dario), physical activities (eg: OneTouch Reveal, Fitbit, Beat Diabetes). Some of the listed applications also have other features such as medication monitoring or providing useful information on diabetes (Shah et al., 2015).

\section{Methodology}

The development environment in which I worked is called Eclipse. It is often used to develop applications written in Java, but in this environment can use other languages like C, C ++, PHP, Prolog and more. 


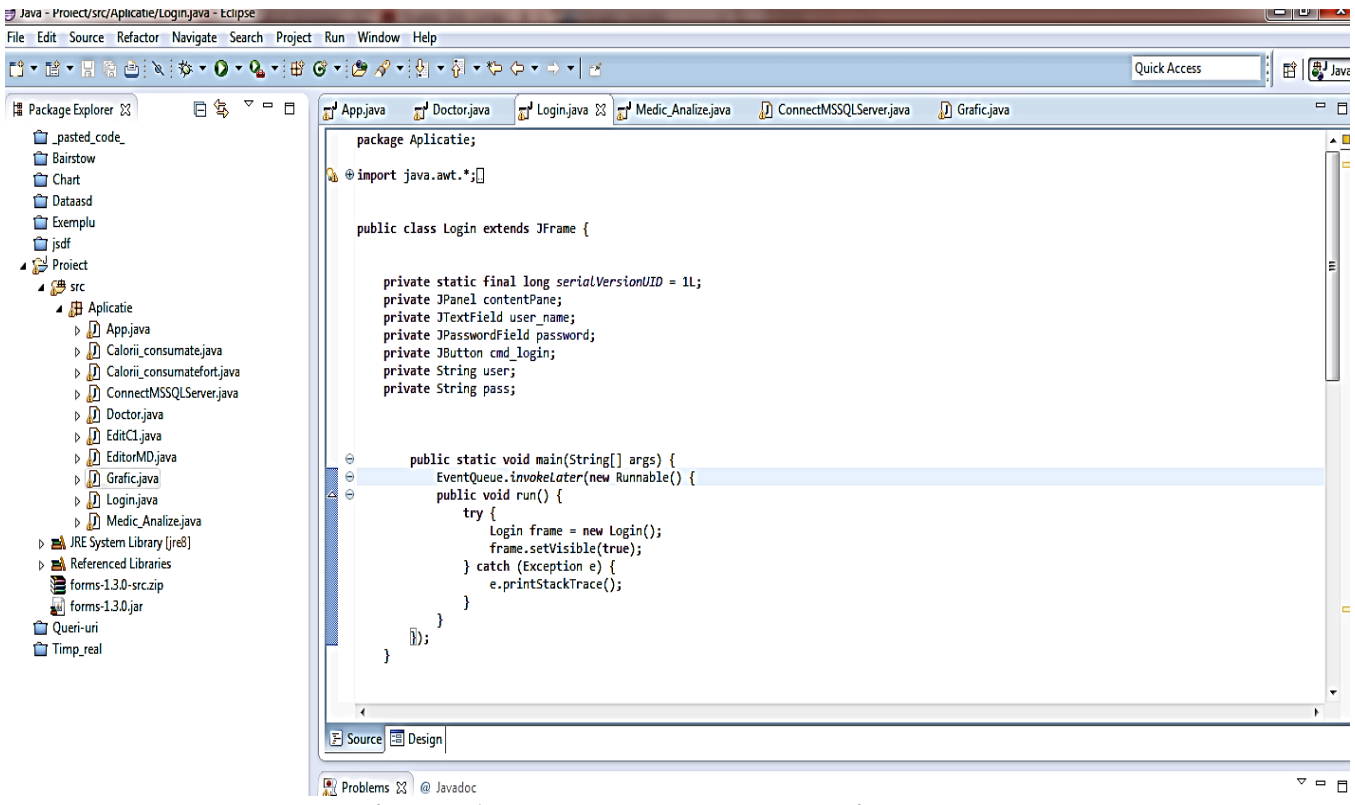

PICBE |

Figure 1. The development environment

Source: Authors' own research.

A major advantage of Eclipse is that it is an open-source platform which means that users can participate in its development or adapt it to their own requirements. The Eclipse architecture also offers an advantage: the integration of extensions (plug-ins) and libraries is easy. This facilitates the integration of various independent software tools.

WindowBuilder is an extension for Eclipse that helps those who want to create interfaces in Java without spending much of the time for the code that creates the buttons and other graphics in the interface. Their functionality can be achieved by writing code.

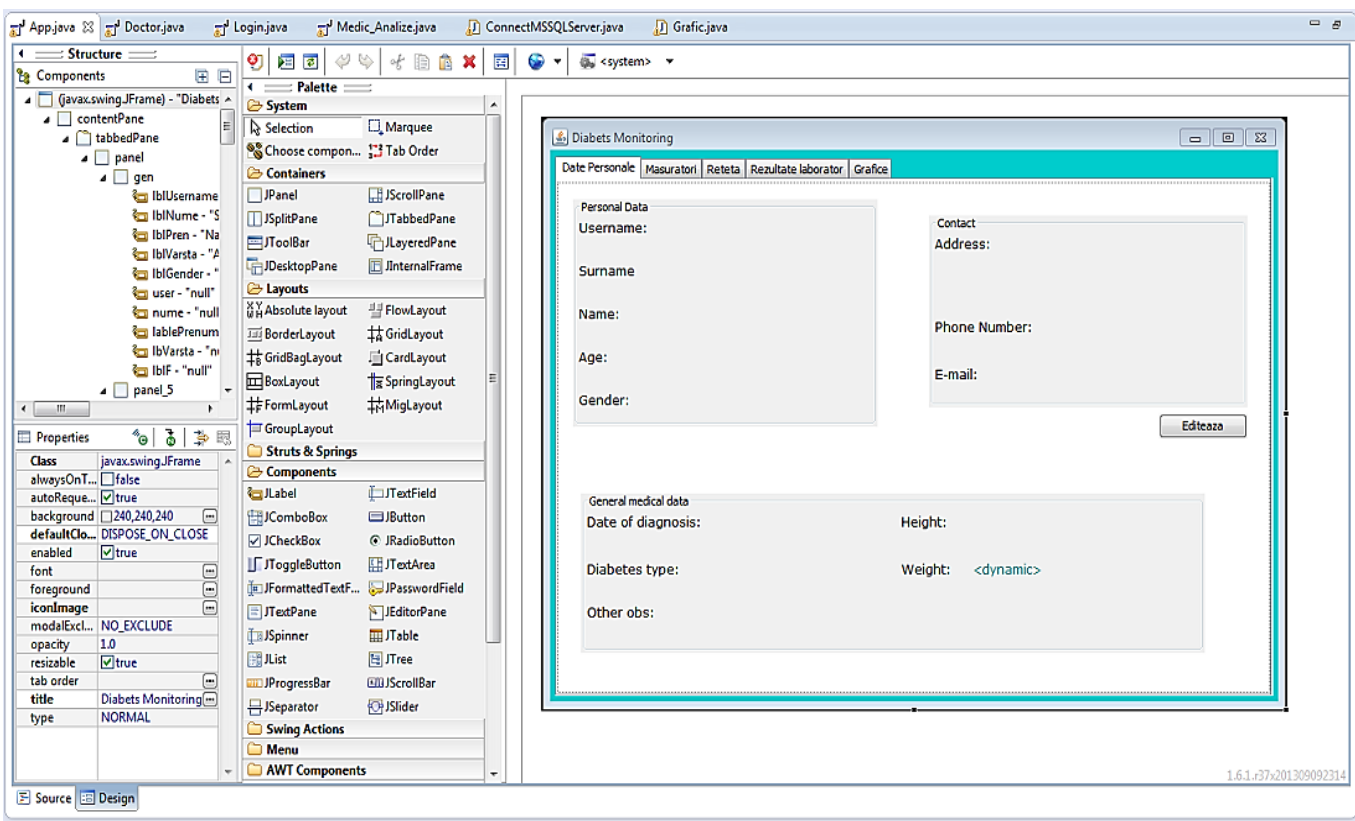

Figure 2. Design View WindowBuilder

Source: Authors' own research.

DOI: $10.2478 /$ picbe-2021-0005, pp. 33-43 , ISSN 2558-9652 |

Proceedings of the $15^{\text {th }}$ International Conference on Business Excellence 2021 
WindowBuilder contains an editor, a parser, views and templates (as can be seen in the Figure 2). The editor is the main component that allows users to create interfaces and contains two tabs: one for editing the design and the other for editing the code.

For certain functionalities that were useful in the development of the application, it was necessary to introduce additional classes that were not offered by the development environment or the WindowBuilder extension. Establishing a connection between a Java application and SQL Server requires additional classes, but also facilities such as a calendar or making a graphic

PICBE | interface. The classes were imported into the Eclipse environment using files with the extension ".jar".

Regarding the data analysis, all the information and data that is circulated between all those who participate as a user in this application, go through the database. In other words, all communication is done through the database, so that any action is recorded, if not in a component table, then in the file that keeps track of database logs.

The component tables contain data that cannot be modified or deleted by users. A user cannot be deleted, the values of the records referring to the name, sex, user cannot be changed by any user, except by the database administrator in exceptional cases.

There is data in certain tables from which real-time reading is essential. They are used for the functionality of the application and are not visible to users (patients, doctors). These data are of the Boolean type (value of 0 or 1), have the role of a traffic light and do not influence patients "measurements, tests and doctors" prescriptions. The value of these fields normally, when no event occurs in the database, is "0". This field is very important for the application because part of it monitors the value of the traffic light (flag) field. When you change data in a table, it also changes its value to " 1 " for the record where the event occurs. The same is done when entering new data in the table, so that after entering the measurements, analyzes or recipes the initial value of the traffic light field is " $1 "$. The role of this field is that when one of the users, doctors or patients, makes a change or introduces new data sets, they are updated in real time in the application interface.

\section{Results and discussions}

The main objective of the paper is to develop a desktop application, through which the patient, doctor and laboratory doctor can connect through a computer with internet access to a database server. This is done according to user authentication, thus defining the level of access to the application or database.

There are three types of users: patient, doctor and laboratory doctor, each having access only to the functions necessary for the role. If the user is a patient, the open window contains six tabs named suggestively: Personal data, Measurements, Prescription, Laboratory results, Graphs, Doctors details.

The first tab that is active is the one with personal data, which contains information about the patient: username, first name, last name, age, sex, contact details (address, phone), medical data (type of diabetes, date of diagnosis, height, weight and other observations). Of all this data, the patient can only change the contact data, having a button at hand to perform this operation.

The "Measurements tab" shows a tool with which the patient may make available personal measurements, certain parameters from which a doctor can monitor it.

The measured data are blood glucose, weight, cholesterol, blood pressure and pulse. These measurements are performed using independent medical instruments.

In addition to these sizes that can be entered, there are also two buttons, each of which opens a calorie calculator. The first button allows the user to calculate the amount of calories based on the 
food consumed. In addition to this value, the values of proteins, carbohydrates and lipids in the same foods are also displayed. The second button opens a computer that offers the ability to view calories burned as a result of the patient's activities. This data is sent to the database at the touch of a button and is thus made available to the doctor who will interpret it.

The next tab, called 'Prescription', contains the indications that the doctor gives to the patient according to his state of health. It offers recommendations for the foods and drinks they can eat, the foods that are forbidden to patients or the maximum amounts that their food can have in some cases. Also, here the doctor prescribes the medication along with its mode of administration, plus other observations (indications) if necessary. The user can see these changes in real time.

The fourth tab "Laboratory results' lists are specified parameters that were examined in the laboratory test results, and each of them are specified normal limits between which should lie, along with units of measurement. Initially, the last analyzes performed in real time are displayed, but the user has the possibility to select a previous date.

The fifth tab from the interface for patients enables them to make a representation as to any measurement (personal or from lab results). Through these graphs the patient can easily understand the situation in which he is or in which direction he is heading. Using the tab is very easy: set the parameter you want to be represented, the time interval in which you want to make the graph, and then by pressing the button the graph is displayed in a new window. It can be resized with the mouse and as many graphics can be displayed at the same time for different parameters.

The last tab shows data about the two types of doctors (general practitioner and laboratory doctor) who have a patient under supervision. It can see data such as name, contact details of doctors (phone, e-mail), and details of his professional training.

If the username is doctor, then a new window will open with six tabs: five similar to those of the patient (having different permissions, except the tab with details about doctors) and in addition to this there is also a tab that acts as a control panel. So, when entering a doctor's data correctly, the first tab that opens in the new window is called the 'Control Panel'. Here is a list of all the patients that the certified doctor has under supervision, the name of the certified doctor and the possibility to upload information about a specific patient. Initially, no data about any patient is loaded in the application, and only after one is selected and the display button is pressed the data is loaded. The following tabs are similar to those for patients, except that doctors have other permissions.

In the tab are presented general information about the patient (name, etc.) doctor can view this data and can not modify data such as contact of the patient, but only general health information.

In the "Measurements" section you will find the data sent by the patient, updated in real time. Here the doctor can only view this data without being able to change it. In the 'Recipes' tab, the doctor sends the information, his recommendations regarding the medication, the foods that can be consumed and that cannot be consumed and the prohibitions for food and drinks. In the fifth tab 'Laboratory results' are displayed as in the patient interface the last results of the analyzes with the possibility to see a history of them, choosing the date on which they were recorded. As in the previous case, the last tab has the same functionality as for the patient. It offers the possibility to graphically represent the evolution over time of parameters measured by both patients and the laboratory.

The latest model of the interface that can be opened is the username corresponding to a doctor performing laboratory analysis and then uploading it to the database is available to doctors and patients to be addressed. This window provides the doctor with three tabs: the control panel, the history of laboratory results and the panel for entering the latest tests. The control panel and the 
history of laboratory results are similar as in the case of the general practitioner who supervises the patient. The last tab offers the possibility to enter the results of the analyzes into the database, and if users are online at the time of uploading them, they are updated in real time.

In order to create the database, the tables and the relationships between the tables certain functional requirements must be considered: patients receive prescriptions (treatment, regimen, medication) from the doctor and tests from the laboratory doctor; patients send personal measurements to the doctor; the measurements are performed once a day (there cannot be two measurements on the same day); the doctor sends prescriptions to the patient; the laboratory doctor loads the results of the analyzes; test results are available to patients and doctors; the doctor may ask the laboratory doctor to perform tests for a specific patient. Based on the above requirements and considering the normalization rules in a database, we have defined the following Table 1.

Table 1. Description of the tables in the database

\begin{tabular}{|c|c|}
\hline Table name & Description \\
\hline Patients & $\begin{array}{l}\text { contains information about patients: general (name, surname), } \\
\text { their contact details (address, telephone, e-mail) and general } \\
\text { medical data (type of diabetes, date of diagnosis, other medical } \\
\text { observations); }\end{array}$ \\
\hline Doctors & $\begin{array}{l}\text { contains information about the doctor: general (name, surname), } \\
\text { information about his training and contact details }\end{array}$ \\
\hline Doctors_Analysis & $\begin{array}{l}\text { contains information about the laboratory doctor: general (name, } \\
\text { surname, age); }\end{array}$ \\
\hline Measurement & contains data (measurements) sent by patients \\
\hline Analysis & contains the results of patient analyzes \\
\hline Recipes & contains prescriptions sent by doctors \\
\hline Patients_Measurements & $\begin{array}{l}\text { contains information about each measurement to which the } \\
\text { patient corresponds and the date on which it was performed }\end{array}$ \\
\hline Patient_Analysis & $\begin{array}{l}\text { contains information about each analysis, to which the patient } \\
\text { corresponds and the date on which it was performed }\end{array}$ \\
\hline Patients_Recepts & $\begin{array}{l}\text { contains information about each prescription, to which the patient } \\
\text { corresponds and the date on which it was made }\end{array}$ \\
\hline Request_Analysis & contains doctors' requests for tests in some patients \\
\hline Calories_Food & contains a list of foods and related nutritional values \\
\hline Calories_Movement & $\begin{array}{l}\text { contains lists of exercises or activities with the values of calories } \\
\text { consumed }\end{array}$ \\
\hline
\end{tabular}

Source: Authors' own research

The application is built on frameworks (JFrame) representing interfaces with users, but access to them varies according to the person trying to log into the application.

The first window to which access is allowed for any type of user (patient, doctor and laboratory doctor) is the authentication window. Its role is to verify that the user is registered in the system and that the username and password have been entered correctly. Also at the authentication level the type of user is determined and depending on the result a new window will open. 
The role of the new frameworks is to limit access only to information that is strictly necessary for each user. This creates an application-level security level for the information in the database.

Any of the windows (frames) that open after authentication contain a number of tabs. Some of these tabs are common to certain users, some are specific to each user, and others are very similar. The differences between the last category are made through small changes (buttons) that offer more facilities to one category of user, compared to another. The number of tabs in each frame is given according to the access level for each user. The tabs can be seen in Figure 3.

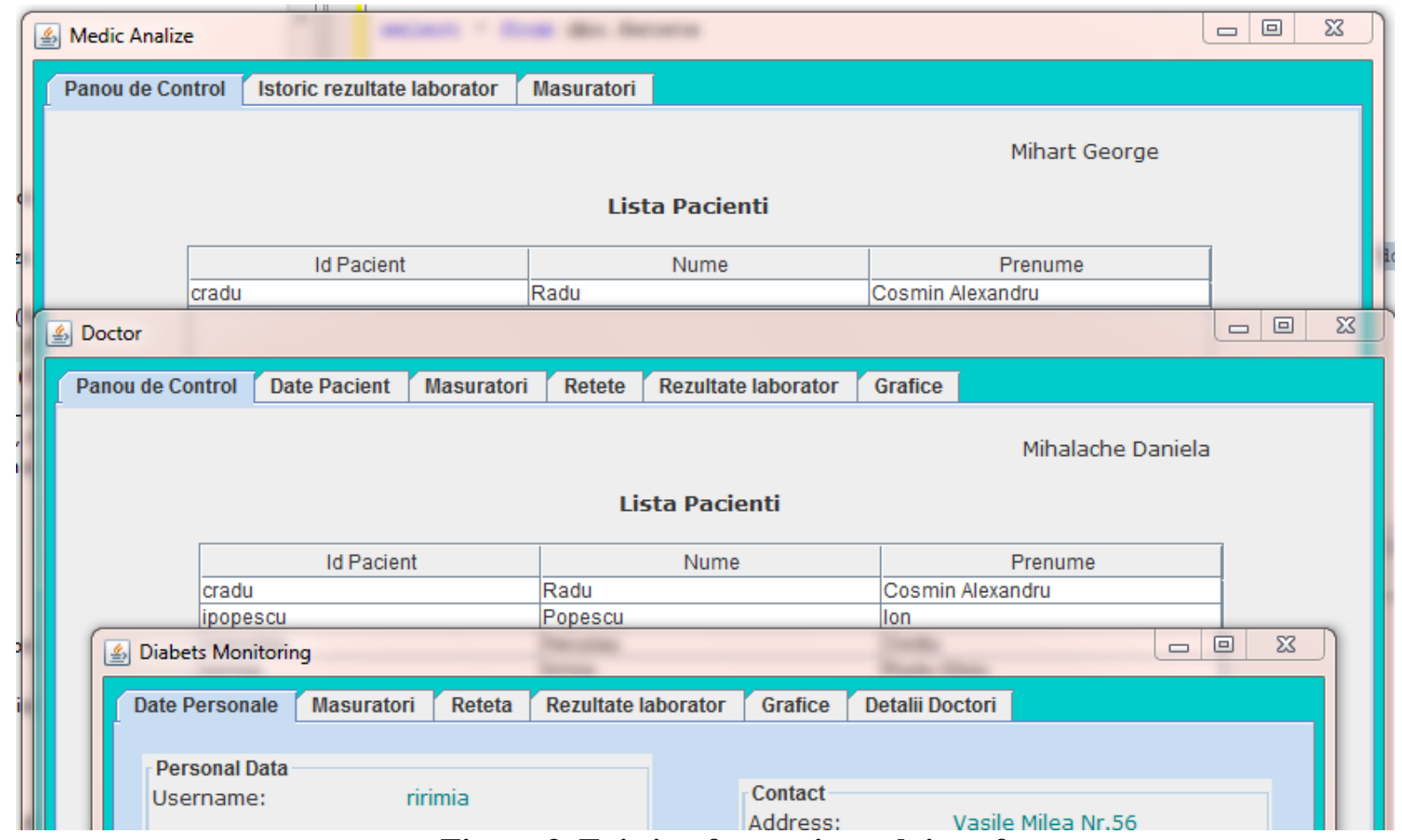

Figure 3. Existing frames in each interface Source: Authors' own research.

The availability of each tab depending on the user and what actions each of them can perform is as follows:

Table 2. Description of each tab in the application

\begin{tabular}{|l|l|}
\hline \multicolumn{1}{|c|}{ Tab name } & \multicolumn{1}{|c|}{ Description } \\
\hline Personal Data or Patient Data & $\begin{array}{l}\text { contains general patient data. It is only available in two of the three } \\
\text { interfaces: Patient and Doctor. Both categories of users can see this } \\
\text { data, but each can only change some of it. }\end{array}$ \\
\hline Measurements & $\begin{array}{l}\text { it is also available to both categories of users, but the interfaces are } \\
\text { different. The patient has the opportunity to enter personal } \\
\text { measurements, to make measurements on the amount of calories of } \\
\text { food consumed and movement performed and send them for } \\
\text { evaluation. Calorie calculators are accessed via two buttons. The } \\
\text { doctor, in this screen has the possibility to analyze the data and to } \\
\text { visualize the date when they were made, but he does not have available } \\
\text { the two buttons for computers, this facility being exclusively offered } \\
\text { to the patient. }\end{array}$ \\
\hline
\end{tabular}




\begin{tabular}{|c|c|}
\hline Tab name & Description \\
\hline Recipes or Recipe & $\begin{array}{l}\text { here patients can only view the last prescription and the date it was } \\
\text { issued. The doctors' interface differs a little, with only a few buttons } \\
\text { needed to analyze old prescriptions and generate new ones. Initially, } \\
\text { the last prescription issued is displayed in the same way as for the } \\
\text { patient, but the doctor also benefits from the option to consult an older } \\
\text { prescription, chosen according to date and a button that generates a } \\
\text { form whose result is the new prescription. }\end{array}$ \\
\hline Laboratory results & $\begin{array}{l}\text { is common to all users. It has the same functionality for all three } \\
\text { frames: displaying the latest analyzes performed and the ability to view } \\
\text { a history of analyzes by date. }\end{array}$ \\
\hline Charts & $\begin{array}{l}\text { visible in the doctor and patient interface. It performs the same } \\
\text { functions and all measured parameters are available for both categories } \\
\text { of patients. So, choose the size and time interval and by pressing a } \\
\text { button draw the desired graph }\end{array}$ \\
\hline Details Doctors & $\begin{array}{l}\text { is only available to patients. Here you will find data about the two } \\
\text { doctors he interacts with. The data are general (name, surname), } \\
\text { contact details (telephone, e-mail) and information on their } \\
\text { professional training. }\end{array}$ \\
\hline Control Panel & $\begin{array}{l}\text { appears only in the interfaces of the two categories of doctors. They } \\
\text { have approximately the same facilities: both have a table in which the } \\
\text { patients they interact with (laboratory doctors) or whom they have } \\
\text { under supervision are displayed, the possibility to choose a patient and } \\
\text { to display his data. The laboratory doctor does not have access to } \\
\text { interfaces where his personal data can be found, he can only view a } \\
\text { history of the analyzes. From this interface the doctor can ask the } \\
\text { laboratory doctor to perform tests for a specific patient by pressing a } \\
\text { button. Only those patients who need to be tested appear in the } \\
\text { laboratory doctor's interface. }\end{array}$ \\
\hline Generate Analysis & $\begin{array}{l}\text { it is available only to the person able to perform this action, namely to } \\
\text { the laboratory doctor. The interface gives him the possibility to enter } \\
\text { the values of the measurements obtained from the tests, and by } \\
\text { pressing the submit button, to write them in the database and update } \\
\text { them in real time in the interfaces of others. }\end{array}$ \\
\hline
\end{tabular}

Source: Authors' own research

Another feature of the application is the ability to graphically represent a measurement or analysis over a period of time.

To choose the time period, I wanted to select it from a calendar, so I imported another library that had these graphic elements defined. I used "JDateChooser", a graphic element through which you can get the desired date in any form. Based on the date to be chosen, the values in the table are selected, and because of this the form we wanted to have is that of SQL (yyyy-mm-dd).

In order to draw the chart and the related graphic elements, it was necessary to import another library and create a new class. The constructor of this class receives as parameters the name of the measurement to be represented, the values and data in the form of vectors. In addition to the class constructor, there is also an implemented method, which transforms the received data into another format and a class from the imported library with functions that fulfill the task of representing the graph. 


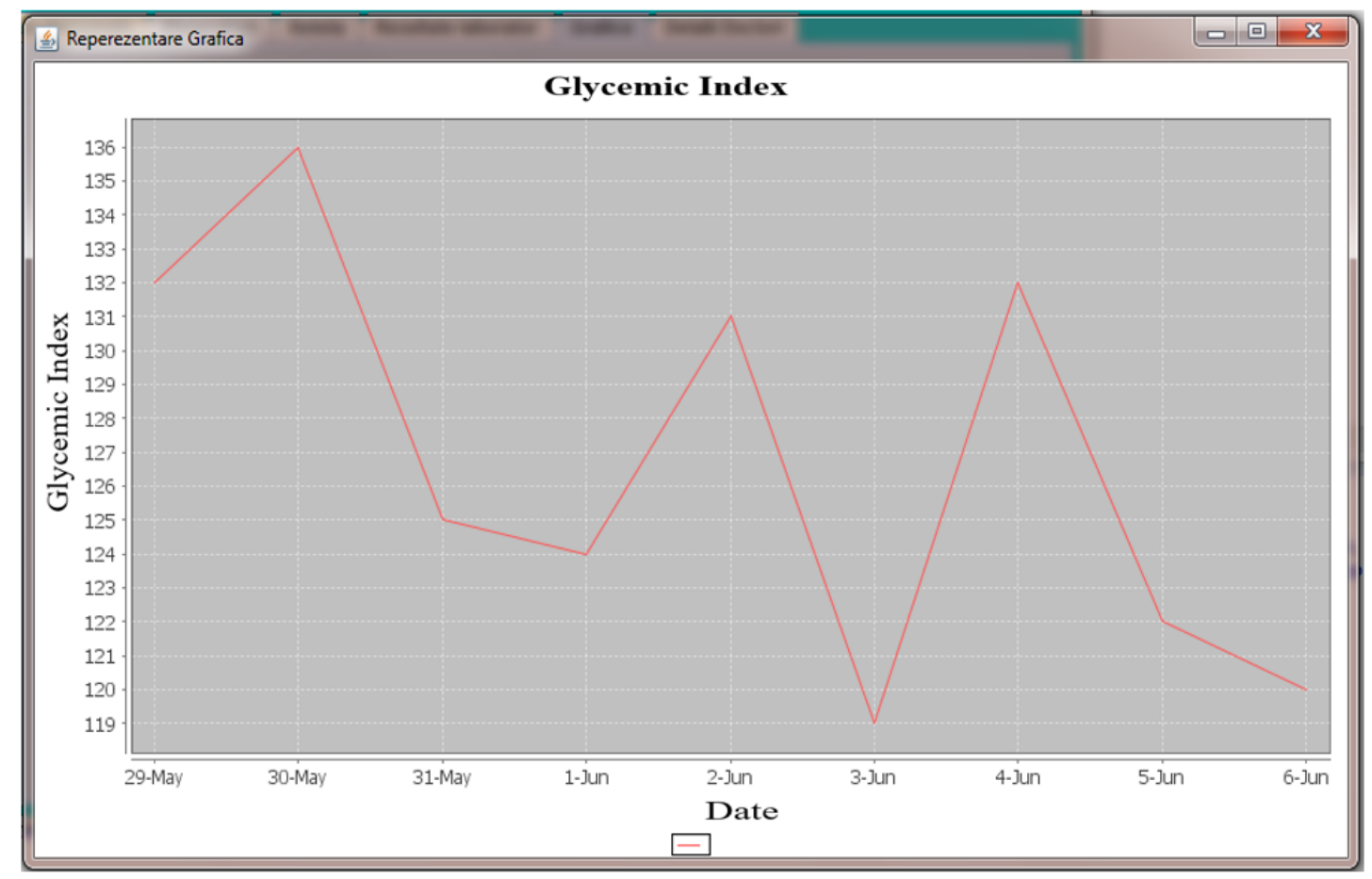

PICBE |

Figure 4. Reprezentarea grafică a unei măsurători intr-o perioada de timp

Source: Authors' own research.

In the control panel in the doctors' interfaces we used a table (JTabel) to display all the patients in the database. The number of records is equal to the number of patients taken from the database. This table was entered in a JScrollPane because it has a predefined size in the tab.

The doctor, in this interface, has the possibility to ask the laboratory doctor if a certain patient performs computer tests. This operation is performed using the "Request_Analysis" table. This table contains the names of the three types of users, the date and a traffic light field (Flag). When the appointment button for a patient is pressed, they are taken from the "Patients" table: the patient's username and the username of the two doctors who have him under supervision. These are entered in the "Request_Analysis" table together with the current date, and in the "Flag" field the value " 1 " is entered.

In the table with the patients in the laboratory doctor's interface, only those patients who have requested for tests are displayed. This means that only those patients with the Flag value "1" are selected from the table. Only after the tests are performed and entered through the interface, the patient is no longer displayed in the laboratory doctor's table. After inserting the results of the analyzes in the tables "Analyzes" and "Patients analysis", performs an update of the field "Flag" in the table "Request_Analysis" with the value "0". Thus the patient no longer meets the selection condition.

Communication between users is done through the database, so it is mandatory that the application reads the data when it changes or new entries appear in the database and they are updated in the interface. Communication with the database in real time cannot be done in a program that has a single thread, in which all instructions are executed in a sequential manner. In our situation, the program can execute the instructions we have presented so far, not the real-time data exchange. One solution in this case is to create a process that runs in parallel. A thread must be implemented to run the data in the background. 
A thread is a sequence of instructions that is executed in a process. Every program has at least one thread inside it. When using threads in an application, it can perform multiple operations at the same time.

In Java there are two methods by which threads can be created: a class is declared as a subclass of the "Thread" class, and this subclass should override the "run ()" method in that class; a class is declared that implements the "Runnable" interface and thus the newly created class can implement the "run ()" method, this being the variant we implemented.

Using multithreading can increase application performance, perform mathematical calculations much faster, and create additional functionality to the application that cannot be performed in a sequential program.

\section{Acknowledgments}

The work of the authors was supported by the project "ANTREPRENORDOC", Contract no. 36355/23.05.2019, financed by The Human Capital Operational Programme 2014-2020 (POCU), Romania.

\section{Conclusion}

The application provides users with a tool through which doctors can monitor the health of patients. This is beneficial in situations where distance is an impediment to the supervision and treatment of people with diabetes. Real-time monitoring was the main topic of interest of this paper. Another objective of the paper is to make the application as well structured as possible, the interface to be intuitive and easy to use and the information circulating between the users of the application to be presented as clearly as possible. Thus, it is possible to ensure the most efficient communication between the participants in this application so that the main beneficiary (patient) receives the best treatments and services. In further development of the application, the first step as the priority should be that it gives the measuring instruments to take over and introduce directly measured data from the patient, without the need to use manual measurements.

\section{References}

Alfian, G., Syafrudin, M., Ijaz, M., Syaekhoni, M.A., Fitriyani, N., \& Rhee, J. (2018). A Personalized Healthcare Monitoring System for Diabetic Patients by Utilizing BLE-Based Sensors and Real-Time Data Processing. Sensors, 18(7).

American Diabetes Association (2019). 7. Diabetes technology: standards of medical care in diabetes - 2019. Diabetes Care, 42(Supplement 1), S71-S80.

Arnold, W. D., Kupfer, K., Little, R. R., Amar, M., Horowitz, B., Godbole, N., San George, R. C. (2020). Accuracy and precision of a point-of-care HbA1c test. Journal of diabetes science and technology, 14(5), 883-889.

Campbell, J. E., \& Newgard, C. B. (2021). Mechanisms controlling pancreatic islet cell function in insulin secretion. Nature Reviews Molecular Cell Biology, 1-17.

Doyle, A. (2020). The Best Diabetes Apps of 2020, Retrieved from https://www.healthline.com/ health/diabetes/top-iphone-android-apps, Accessed on 1.02.2020.

International Diabetes Federation, (2020), What is Diabetes, Retrieved from https://www.idf.org/ aboutdiabetes/what-is-diabetes.html, Accessed on 6.02.2020.

Jimenez, G., Lum, E., \& Car, J. (2019). Examining diabetes management apps recommended from a Google search: content analysis. JMIR mHealth and uHealth, 7(1). 
Petrie, J. R., Guzik, T. J., \& Touyz, R. M. (2018). Diabetes, hypertension, and cardiovascular disease: clinical insights and vascular mechanisms. Canadian Journal of Cardiology, 34(5), 575-584.

Shah, V. N., \& Garg, S. K. (2015). Managing diabetes in the digital age. Clinical Diabetes and Endocrinology, 1(1), 1-7. 